\title{
ABSTRACTS \\ Meeting of \\ The American Pediatric Society and The Society for Pediatric Research
}

\author{
Atlantic City, New Jersey, April 26-29, 1967
}

Special Sections

(APS) Paper submitted to The American Pediatric Society

(SPR) Paper submitted to The Society for Pediatric Research

Demonstration of Coupled Oxidative Phosphorylation in the Fetal Heart. Joseph B. Warshaw*, Dept. of Bioenergetics Res., Retina Foundation and the Dept. of Pediatrics, Harvard Med. Sch., Boston, Mass. (introduced by H. Haessler).

It has been recognized that newborn and fetal mam mals exhibit greater resistance to the effects of anoxia than adults. This has been attributed to the prominence of glycolysis as an energy yielding pathway in early life. Since the efficiency of oxidative phosphorylation in the developing heart has not been assessed previously, the present investigation was undertaken. Heart mitochondria were isolated from the bovine fetus estimated by the crown-rump length and weight to range from 65 to 160 days gestational age. Mitochondrial efficiency was determined from $\mathrm{ADP} / \mathrm{O}$ ratios and respiratory control values. Substrates entering the two flavoprotein limbs of the respiratory chain were utilized. ADP/O ratios obtained with glutamatemalate, a DPNH linked substrate, were above two in all and approached 3 in the five animals in the younger (65 to 80 day) group. With succinate as the substrate values were above one with many approaching two. Thus for both substrates ratios were in the normal adult range. All preparations exhibited respiratory control values considered normal for mitochondria isolated from the mature heart (average ratios above 6 ). Specimens oxidized the substrates readily with no apparent age relationship. Under the conditions of the isolation procedure employed, mitochondrial yields from the fetal hearts were generally half of those from the adult tissue. These preliminary data indicate that the machinery of oxidative phosphorylation in the developing heart is intact and functionally similar to that of the adult. (Supported in part byMassachusetts Heart Association) (SPR)

Acute and Chronic Effects of one Umbilical Artery Ligation in the Lamb Fetus. Georg EmmanoulliDes*, Duane Townsend*, Robert Bauer* and Robert Perens*, U.C.L.A. School of Med. and Harbor General Hosp., Torrance, Calif. (introduced by Joseph.W.Sr. Geme, Jr.).

* By invitation
We have studied some of the acute and chronic effects of the ligation of one umbilical artery in the lamb fetus. Via laparotomy and hysterotomy one umbilical artery is isolated. A polyvinyl catheter is advanced retrogradely into the fetal aorta. The distal portion of the artery is ligated, and the catheter exteriorized. Arterial blood pressure, heart rate, and blood $\mathrm{pH}, \mathrm{pCO}_{2}$ and $\mathrm{pO}_{2}$ determinations were performed at various intervals until the end of the gestation.

Preliminary observations indicate that fetal survival is possible. Although fetuses near term usually do not survive this insult more than a few hours, long term survival (4 to 57 days) was observed in 6 animals of an earlier gestational age ( 80 to 120 days). After an initial period of hypoxia, hypercapnia, and acidosis, a remarkable stabilization occurred and 'normal' values were observed. Mean arterial pressures ranged from 48 to $66 \mathrm{~mm} \mathrm{Hg}$ and heart rates from 140 to 260 per minute. The range of arterial $\mathrm{pH}$ was 7.37 to 7.45 , $\mathrm{pO}_{2} 11$ to $24 \mathrm{~mm} \mathrm{Hg}$ and $\mathrm{pCO}_{2} 34$ to $53 \mathrm{~mm} \mathrm{Hg}$. In spite of this apparently normal gas and hydrogen ion exchange between mother and fetus, profound fetal malnutrition has been observed. This 'experimental model' may have a potential value for studying chronic fetal distress due to placental insufficiency. (SPR)

3 Hypoxemia and Protein Clearance from the Pulmonary Vascular Bed of Pups. URABALA BoONYAPRAKOB*, Paul Taylor, Doris Watson*, Viera WaterMAN* and Eugene Lopata*, Dept. of Ped., Univ. of Pittsburgh, and Magee-Womens Hosp., Pittsburgh, $\mathrm{Pa}$.

Clearances of plasma proteins from the pulmonary circulation (CI prot.) are 2-3 fold greater for 2-3 weekold pups than for adult dogs (J.Pediat. 69: 966, 1966). Those data probably indicate greater permeability of the pulmonary capillaries to plasma proteins (PCP) for pups than for adult dogs as transpulmonary artery wedge pressure and effective pulmonary blood flow were similar for pups and adult dogs. The effect of hypoxemia on $\mathrm{Cl}$ prot. has now been evaluated in five anesthetized, normothermic, artificially ventilated 2-3 week-old pups. Gl prot. was calculated from lung lymph and serum protein concentrations and lung lymph flow rate and expressed as $\mu$ l plasma cleared/ $100 \mathrm{~g}$ lung/h. During $30 \mathrm{~min}$ ventilation with $10 \% \mathrm{O}_{2}$, 